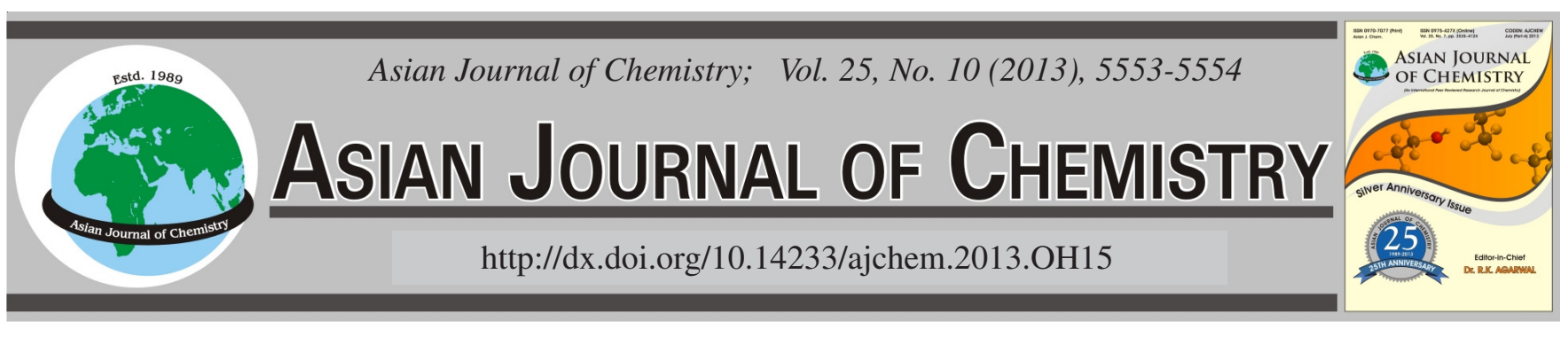

\title{
Facile Synthesis of Super-hydrophobic CuO Film on Copper Substrate $†$
}

\author{
Rongchun NiE ${ }^{1, *}$, ChuYang $\mathrm{XU}^{2}$, Yanfen $\mathrm{W}_{\mathrm{AnG}}{ }^{2}$ and BenXIA $\mathrm{LI}^{2}$
}

${ }^{1}$ School of Chemical Engineering, Anhui University of Science and Technology, Huainan 232001, Anhui Province, P.R. China

${ }^{2}$ School of Materials Science and Engineering, Anhui University of Science and Technology, Huainan 232001, Anhui Province, P.R. China

*Corresponding author: Fax: +86 554 6668643; Tel: +86 554 6634072; E-mail: rchnie@ aust.edu.cn

\begin{abstract}
Super-hydrophobic metal films have attracted significant attention due to their many advantage and wide applications. In the present work, we report a convenient one-step route for a large scale of butterfly-like or flower-like $\mathrm{CuO}$ microstructures constructed from nanoplates on copper substrate at low tempreture. After octadecane thiol hydrophobization, the film exhibited excellent super-hydrophobicity and little sliding angle, which could be attributed to combination of the improved dual scale roughness of $\mathrm{CuO}$ and the octadecane thiol coating with low surface energy. The super-hydrophobic metal coating may be applicable for multiple fields such as self-cleanning or anticorrosion field.
\end{abstract}

Key Words: CuO, Super-hydrophobic, Octadecane thiol, Metal surface.

\section{INTRODUCTION}

Recently, super-hydrophobic surfaces with the water contact angle greater than $150^{\circ}$ have attracted researchers' interests because of their excellent water-proof property and potential application as self-cleaning products, such as textiles, building coating, separation and filtration ${ }^{1-4}$. It is well known that the geometrical structure of the surface and the use of low-surface-energy materials may be two key factors in realizing the super-hydrophobicity of solid surfaces ${ }^{5}$. Inspired by it, various methods have been developed to prepare superhydrophobic surfaces on different substrates, including the electrodeposition, chemical etching, sol-gel method, chemical vapour deposition method, electrospinning method, etc. ${ }^{6}$. These super-hydrophobic surfaces based on polymers, metals, glasses, semiconductors and carbon nanotubes have been fully reported. However, it may be significant to study superhydrophobic surfaces by a simple and economic method in order to make practical application in industrial field ${ }^{7}$.

In general, super-hydrophobic surfaces can be prepared in two ways: one is constructing the rough structure on the hydrophobic material surface and the other is coating low surface energy materials on the rough surface. Herein, we presented a simple, inexpensive and time-saving method for fabricating stable super-hydrophobic $\mathrm{CuO}$ surfaces on copper substrate by immersing and modifying copper foil method at low temperature.

\section{EXPERIMENTAL}

A typical synthesis of hierarchical $\mathrm{CuO}$ microstructures on copper foil was carried out as follows. Firstly, a copper foil $(15 \mathrm{~mm} \times 50 \mathrm{~mm} \times 0.15 \mathrm{~mm})$ was ultrasonically washed in dilute $\mathrm{HCl}$ solution for $20 \mathrm{~min}$ and subsequently washed several times in deionized water and absolute ethanol before use. The treated copper foil was placed in a sealed jar $(50 \mathrm{~mL})$ containing a mixed solvent of distilled water $(10 \mathrm{~mL})$ and ammonia $(10$ $\mathrm{mL}$ ) and then left still at $80^{\circ} \mathrm{C}$ for $24 \mathrm{~h}$. After the reaction, a dark film on copper foil was taken out from the reaction mixture, washed and dried well for further characterizations. Then, the obtained $\mathrm{CuO}$ film sample was immersed in an ethanol solution of octadecane thiol with constant stirring for $30 \mathrm{~min}$ at $60{ }^{\circ} \mathrm{C}$ to complete a self-assembly process.

The X-ray diffraction (XRD) were recorded with Shimadzu XRD-6000 with $\mathrm{CuK}_{\alpha}$ radiation. The scanning electron microscopy (SEM) was performed on Rili S-3000N. The contact angles of the thin-films were measured with a contact angle meter C20 (Kono, America).

\section{RESULTS AND DISCUSSION}

Fig. 1 shows the XRD pattern of the as-obtained $\mathrm{CuO}$ sample. It can be seen that all of the observed peaks can be indexed to a pure monoclinic phase $\mathrm{CuO}$ (JCPDS card, 05-0661) in the pattern. Additionally no peak for other types 


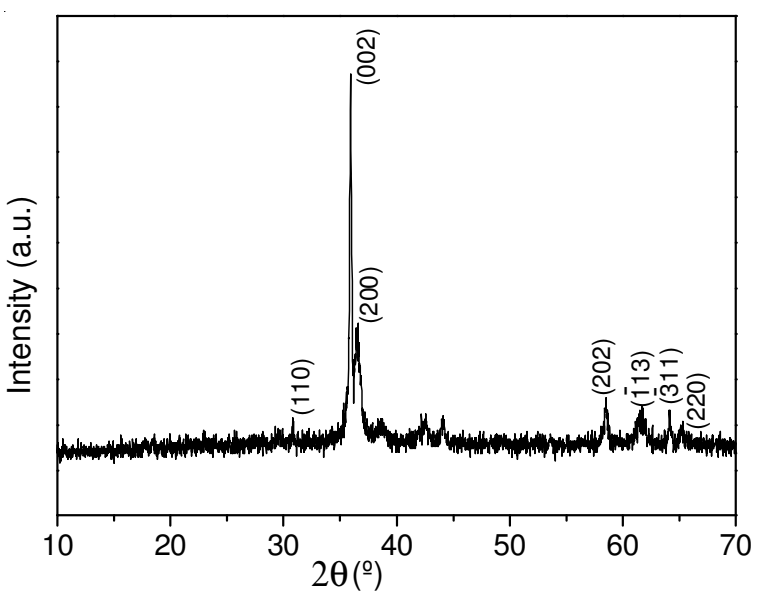

Fig. 1. XRD pattern of the as-obtained $\mathrm{CuO}$ sample

of $\mathrm{Cu}(\mathrm{OH})_{2}$ or $\mathrm{Cu}_{2} \mathrm{O}$ was observed, indicating the high purity and well crystallinity of the sample.

The morphology of the as-prepared product on copper foil surface is observed by SEM. Fig. 2 shows SEM images of the $\mathrm{CuO}$ sample at different magnifications. From the representative low-magnification SEM image shown in Fig. 2a, it is observed that the whole surface of copper foil has been covered with a mass of compact $\mathrm{CuO}$ particles. A high enlargement SEM image of the $\mathrm{CuO}$ product is presented in Fig. 2b. It reveals that these particles are constructed from many irregular nanosheets to form butterfly-like or flower-like mixed microstructures. There are many small submicro or nanometer gaps within these microstructures, which can capture or absorb effectively a lot of air, resulting in the air/water interface greatly increased.
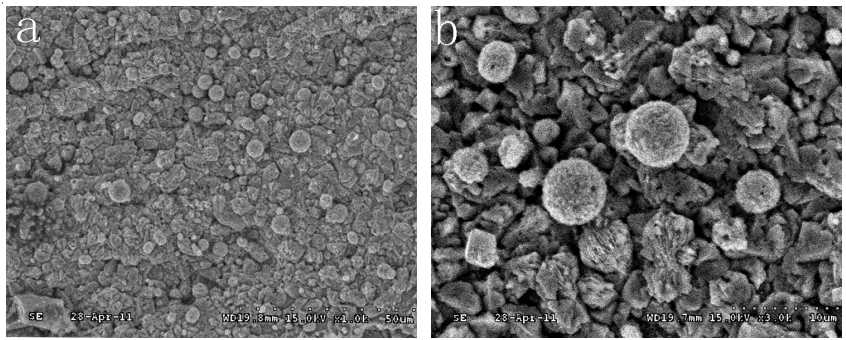

Fig. 2. SEM images of the $\mathrm{CuO}$ sample at different magnifications

The surface wettability of the as-prepared films has been evaluated by the water contact angle measurement. As shown in Fig. 3a, the contact angle of the film surface is measured to be $13^{\circ}$ before the treatment of copper foil, showing the hydrophilic property. Therefore, we modified $\mathrm{CuO}$ micro/ nanostructures by octadecane thiol to decrease surface energy. Fig. $3 \mathrm{~b}$ shows that the water contact angle of the film is measured to be $152^{\circ}$ after the dipping treatment with lowsurface-energy octadecane thiol, exhibiting the remarkable super-hydrophobicity. If the film was slightly tilted, the water droplets would roll down, indicating a very little sliding angle.

In general, the wettability of solid surface is related to surface topography and modification with low-surface-energy material. Increasing surface roughness and lowering surface energy can remarkably enhance surface water repellency. In our reactive system, when the copper foil was immersed into b

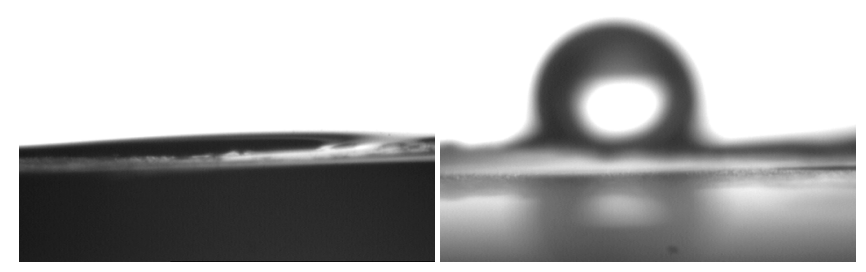

Fig. 3. Static photograph of water contact angle (a) bared copper foil without modification, (b) resultant $\mathrm{CuO}$ film after octadecane thiol modification

octadecane thiol solution, a layer of single thiol molecule hydrophobic coating will be formed on the copper foil surfaces. It can decrease the surface free energy effectively, inducing the contact angle of the surface greatly enhanced. In addition, the surface with micro/nano dual scale roughness provides the necessary structural condition for the super-hydrophobicity. Therefore, the generation of $\mathrm{CuO}$ with micro/nano dual scale roughness and the modification with low-surface-energy octadecane thiol play a key role for the super-hydrophobicity. Fig. 4 shows the wettability diagram of the copper foil surface.

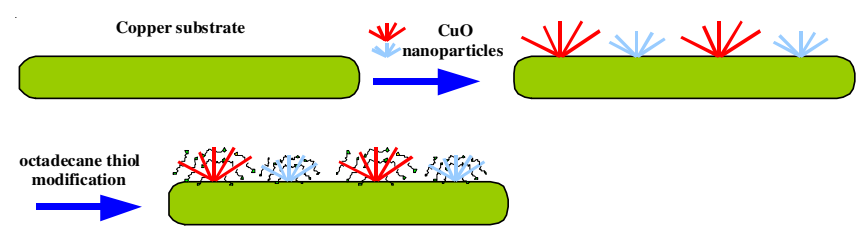

Fig. 4. Wettability diagram of the copper foil surface

\section{Conclusion}

We have presented a simple method of fabricating super-hydrophobic surface of octadecane thiol modified $\mathrm{CuO}$ micro/nanostructure film with on copper substrate at low tempreture. Combining the surface topography provided by the $\mathrm{CuO}$ dual scale roughness at micro/nanometer scales and the low-surface-energy layer formed by octadecane thiol adsorption render the coating super-hydrophobicity. This method may be used to fabricate other metal surfaces with super-hydrophobicity at a low cost.

\section{ACKNOWLEDGEMENTS}

The work was financially supported by Natural Science Research for Colleges and Universities of Anhui Province of China (Grant No. KJ2010A101) and the Natural Science Foundation of Anhui Province (10040606Q15).

\section{REFERENCES}

1. W.X. Hou and Q.H. Wang, Langmuir, 25, 6875 (2009).

2. Y.L. Zhou, M. Li, B. Su and Q.H. Lu, J. Mater. Chem., 19, 3301 (2009).

3. X.M. Li, D. Reinhoudt and M. Crego-Calama, Chem. Soc. Rev., 36, 1350 (2007).

4. L.B. Zhang, H. Chen, J.Q. Sun and J.C. Shen, Chem. Mater., 19, 948 (2007).

5. Y.F. Wang, B.X. Li and C.Y. Xu, Superlattices Microstruct., 51, 128 (2012).

6. J.T. Wang, X.H. Zhang, H. Wang and X.M. Ou, Acta Phys.-Chim. Sin., 27, 2233 (2011).

7. F.Y. Xu, L.J. Liu, J. Qin, B. Liu and S. Mei, Acta Phys.-Chim. Sin., 28, 693 (2012). 